\title{
Revista

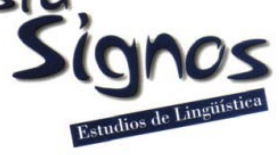

\section{Diferencias en las creencias entre hombres y mujeres acerca del aprendizaje del idioma inglés ${ }^{1}$}

\section{Differences between males and females' beliefs about English language learning}

\author{
J uan Fernando Gómez Paniagua \\ TECNOLÓgico de ANTIOQUia - INSTITUCIÓN UniVERSITARIA \\ COLOMBIA \\ juanfer777@gmail.com
}

Recibido: 21-IX-2016 / Aceptado: 18-VIII-2017

\section{Resumen}

El propósito del presente estudio es describir las diferencias entre las creencias de hombres y mujeres acerca del aprendizaje del idioma inglés, lo cual se alcanzó mediante la descripción de dichas creencias en el contexto del Tecnológico de Antioquia Institución Universitaria (Colombia). La investigación es de tipo descriptiva; bajo un diseño no experimental, de campo, transeccional descriptiva. La población estuvo conformada por un total de 266 estudiantes. Se empleó el instrumento especializado BALLI (Inventario de Creencias sobre el Aprendizaje de un Idioma), conformado por un total de cinco cuestionarios que fueron analizados con estadística descriptiva e inferencial empleando el software SPSS. Los hallazgos indicaron que, en general, ambos géneros tuvieron creencias similares frente al aprendizaje del inglés en las cinco dimensiones del BALLI. Sin embargo, el estudio reveló que las mujeres sienten mayor inseguridad para comunicarse, piensan que los niños tienen habilidades especiales y que la tipología sintáctica del español es diferente a la del inglés. Por otro lado, los hombres son escépticos a que en su país de origen puedan adquirir la competencia comunicativa; sienten una mayor motivación instrumental y manifiestan que el aprendizaje del inglés no es una habilidad exclusiva de ciertas personas.

Palabras Clave: Competencia comunicativa, creencias sobre el idioma, desarrollo de habilidades, diferencias entre sexos, métodos de aprendizaje. 


\begin{abstract}
The current research aims at describing the differences between males and females' beliefs about English language learning. Such a purpose was achieved by means of describing the gender differences about language learning at Tecnológico de Antioquia Institucion Universitaria (Colombia) setting. This corresponds to a descriptive research carried out under a non-experimental design, transactional descriptive field. The representative sample of this study was comprised of 266 students. Data were collected through the specialized BALLI instrument (Beliefs about Language Learning Inventory) comprised of five questionnaires that were analyzed with descriptive and inferential statistics by means of the SPSS software. Findings indicated that, overall, both genders held similar beliefs about language learning in the whole BALLI dimensions. Nevertheless, this study also indicated that females feel less confident to speak, think that children have special abilities, and consider that the language typological system is different in both English and Spanish languages. On the other hand, males are not convinced that they can achieve the communicative competence in their country of origin, feel a higher instrumental motivation, and believe that learning a foreign language deals with certain abilities that some people have.
\end{abstract}

Key Words: Communicative competence, language beliefs, skills development, gender differences, learning methods.

\title{
INTRODUCCIÓN
}

Con relación a los procesos de enseñanza y aprendizaje del idioma inglés, los estudiantes tienen ciertas creencias que facilitan o dificultan su aprendizaje. En este sentido, Cohen (2010) afirma que las creencias que tienen los estudiantes sobre el aprendizaje de un idioma son determinantes para su progreso y sostiene que algunas de ellas están relacionadas con la motivación. En el aprendizaje de una lengua extranjera hay ciertos factores que influyen directamente en el logro de tal propósito. Desde la perspectiva de Horwitz (1999), estos se refieren a las estrategias que usan los estudiantes, al grado de motivación, los rasgos de la personalidad, el contexto, los estilos de aprendizaje, el nivel del idioma, la edad, el sexo y las creencias que tengan frente al aprendizaje. En el Tecnológico de Antioquia-Institución Universitaria, se observan estudiantes de diferentes niveles que tienen dificultades para alcanzar la competencia comunicativa, y es posible que este hecho esté ligado a las creencias que poseen con relación al aprendizaje del inglés. Actualmente se presenta un bajo rendimiento académico en el área de inglés en los diferentes programas técnicos, tecnológicos y profesionales de la institución. Esta afirmación se sustenta en los resultados suministrados por la oficina de Admisiones y Registro donde se evidencia que el 31,6\% del total de los estudiantes que finalizaron los cursos entre los niveles uno y seis, reprobaron la asignatura debido a que no lograron las competencias requeridas en el nivel correspondiente.

Por otro lado, los estudiantes manifiestan tener ciertas creencias frente al aprendizaje del inglés, las cuales poco favorecen el desarrollo de la competencia 
comunicativa: 'la parte más importante del inglés es la gramática', 'para aprender inglés hay que ser muy inteligente', 'solo se aprende inglés si se viaja a un país de habla inglesa', 'hablar inglés es como hablar español al revés', 'el inglés es el idioma más difícil de aprender', 'si uno no pronuncia bien es mejor no decir nada', entre otras. Estas creencias fueron obtenidas de acuerdo con las evaluaciones suministradas por los docentes al finalizar el semestre académico.

Por lo anterior, se tiene la convicción que conocer las creencias de los estudiantes sobre el aprendizaje de un idioma extranjero podría ayudar a los docentes a tener una mayor comprensión sobre aquellos aspectos que dificultan dicho proceso de aprendizaje.

En concordancia con las situaciones planteadas, el propósito de esta investigación fue describir las diferencias entre hombres y mujeres acerca del aprendizaje del idioma inglés, lo que indica que la variable se enmarca en el sexo masculino y femenino. Desde la perspectiva de Ellis (1994), el término sexo indica una distinción biológica y el género se refiere a una distinción social. En este estudio, se empleará el término sexo para referirse a la distinción biológica entre hombres y mujeres.

El artículo tiene la siguiente estructura: en la primera sección se presenta el marco teórico de la investigación; en la segunda se expone el marco metodológico y se describen los cuestionarios referentes a cada dimensión BALLI (Inventario de Creencias sobre el Aprendizaje de un Idioma); en la tercera parte se presentan los resultados y se indican los ítems donde se encontraron diferencias significativas entre ambos sexos; en el cuarto apartado se realiza la discusión de los resultados y en el quinto se plantean las conclusiones, las líneas futuras de investigación y las limitaciones.

\section{Marco teórico}

\subsection{Creencias sobre el aprendizaje de una lengua extranjera}

Victori y Lockhart (1995) sustentan que las creencias corresponden a suposiciones que los estudiantes tienen sobre los factores que hacen parte de su proceso de aprendizaje, sobre la naturaleza del lenguaje y sobre ellos mismos desde una perspectiva de sujetos que están aprendiendo un idioma. Al respecto, Gaete, Gómez y Benavides (2017) afirman que en la actualidad las creencias de estudiantes, docentes y padres de familia se han convertido en un tema de investigación en el ámbito educativo.

Para Borg (2011), las creencias tienen que ver con proposiciones que las personas presumen ciertas, contienen un aspecto afectivo y muestran cierta resistencia a ser modificadas. Por su parte, Breen (2014) sustenta que las creencias son un aspecto determinante en el proceso de aprendizaje y están relacionadas con las experiencias 
vividas por cada sujeto. En su trabajo sobre estilos de aprendizaje y formación bilingüe (Loaiza \& Galindo, 2014) explican que el bilingüismo requiere ser abordado desde una perspectiva antropológica, dado que el uso del lenguaje comprende conocimientos, ideas y creencias que un grupo de personas comparte.

En el contexto latinoamericano, Ormeño y Rosas (2015), identificaron que las creencias que generan mayores barreras en el aprendizaje del inglés tienen que ver con las dimensiones de la naturaleza del aprendizaje del idioma y las estrategias de comunicación. Por su parte, Díaz Larenas y Moralez Campos (2015) indicaron que los estudiantes de los niveles inferiores que fueron analizados evidenciaron tener creencias más positivas frente al estudio del idioma, con relación a su utilidad en la sociedad. Los grados superiores reportaron tener experiencias negativas y se mostraron más críticos respecto a las clases. Gómez (2017) encontró que ciertas creencias relacionadas con las dimensiones de la naturaleza del aprendizaje del inglés y las estrategias de aprendizaje representan grandes retos para los estudiantes.

Desde la perspectiva de Dörnyei (2005) hay una gran variedad de estudios que corroboran que la edad y el sexo son variables de gran importancia en cuanto al aprendizaje de una lengua extranjera. En cuanto a la creencia que las mujeres aprenden idiomas 'mejor' que los hombres, Oxford, Nyikos y Ehrrnan (1988) declaran que es un campo que poco se ha investigado desde la perspectiva de las estrategias de aprendizaje que emplean los estudiantes para mejorar su aprendizaje.

Con relación al aprendizaje del vocabulario, el estudio de San Mateo Valdehíta (2015) confirmó que la estrategia de escritura permite a las mujeres memorizar un mayor número de palabras, aunque también se evidenció que no hay una diferencia significativa entre ambos sexos en el desarrollo de tareas de selección de definiciones y de ejemplos. En la investigación realizada por Phakiti (2003) sobre diferencias entre hombres y mujeres en la comprensión lectora, se concluyó que los hombres emplean más estrategias metacognitivas que las mujeres y no se presentaron diferencias entre ambos sexos en cuanto al uso de estrategias cognitivas. Frente al uso de estrategias para el aprendizaje del inglés, Radwan (2011) definió que los hombres emplearon más estrategias sociales.

De acuerdo con Zeynali (2012), el sexo es una variable que afecta el uso del idioma y la manera de aprenderlo ya que se presentan diferencias biológicas, psicológicas y socioculturales entre hombres y mujeres. En este sentido, López (2006) indica que hay cierta relación entre el género, el éxito y el aprendizaje de una lengua extranjera, la cual está determinada por factores neurológicos, cognitivos, afectivos, sociales y educativos. 


\subsection{Estudios sobre el sexo masculino y femenino desde el instrumento BALLI}

El instrumento BALLI propuesto por Horwitz (1987) se constituye en el de mayor prestigio (Barrios, 2014), y ha marcado un hito en la investigación de creencias de los estudiantes sobre el aprendizaje de una lengua extranjera (Ormeño \& Rosas, 2015). Su mayor ventaja radica en que está dividido en las dimensiones que comprenden el proceso de aprendizaje de una lengua extranjera y cada ítem puede ser adaptado a un contexto determinado. Su pertinencia es sustentada en las investigaciones realizadas en diferentes lugares del mundo: Hungría (Rieger, 2009); Irán (Barkhordar \& Rastegar, 2012); Chile (Ormeño \& Rosas, 2015), entre otros.

El instrumento consta de 34 creencias, agrupadas en cinco dimensiones (Horwitz, 1987):

A. Aptitud para las lenguas extranjeras (8 ítems): este cuestionario contiene las creencias sobre el éxito o fracaso que alguien pueda tener frente al aprendizaje de un idioma.

B. La dificultad del aprendizaje de una lengua extranjera (5 ítems): esta dimensión corresponde al grado de dificultad del idioma y el tiempo requerido para su aprendizaje.

C. La naturaleza del aprendizaje de lenguas (7 ítems): estas creencias están relacionadas con la estructura del idioma, aspectos como el acento, la cultura y el grado de dificultad que tiene cada una de las habilidades comunicativas.

D. Las estrategias de aprendizaje y comunicación (8 ítems): estos ítems se refieren a las estrategias que tienen las personas para aprender un idioma: inferir significados, autocorrección, repetición, aprendizaje de vocabulario y gramática, memorización.

E. Motivaciones y expectativas (6 ítems): esta dimensión contiene las creencias sobre la importancia de aprender un idioma extranjero y la manera en que dicho aprendizaje ayudará a conocer personas nativas del idioma y posibilitará obtener un buen empleo.

En cuanto a estudios relacionados con las diferencias entre ambos sexos acerca del aprendizaje del idioma inglés, Bernat y Lloyd (2007) utilizaron el BALLI con una población de 155 mujeres y 107 hombres en el contexto de Australia. Se encontró que, en general, las mujeres y los hombres tienen creencias similares en cuanto al aprendizaje de una lengua extranjera, a excepción del ítem correspondiente a la inteligencia y a la práctica del inglés con hablantes nativos. Se concluyó que las mujeres, en una mayor proporción que los hombres, consideraron que la inteligencia es un factor importante en el aprendizaje de un idioma y que ellas disfrutan más hablar en inglés con otros australianos.

Por su parte, Rieger (2009) condujo un estudio con 86 mujeres y 23 hombres quienes estudiaban alemán e inglés en la Universidad de Budapest. Se analizaron las 
cinco dimensiones con una versión del BALLI adaptada al contexto de Hungría. Los resultados evidenciaron que hay diferencias considerables en las creencias frente al aprendizaje de una lengua extranjera según el género; estas hacen referencia a las técnicas y estrategias aplicadas para aprender el idioma y el grado de dificultad.

Por otro lado, Daif-Allah (2012) realizó un estudio cuyo propósito fue explorar las creencias de los estudiantes de inglés de la Universidad de Quassim (Arabia Saudita) y establecer los posibles efectos del sexo en dichas creencias. Se conformaron dos grupos para esta investigación: 125 hombres y 111 mujeres. Los resultados indicaron que ambos sexos tuvieron creencias similares en cuanto a la dificultad y la naturaleza del idioma. También se presentaron diferencias significativas en las dimensiones relacionadas con la motivación y expectativas, la actitud y las estrategias de comunicación.

En el contexto iraní Barkhordar y Rastegar (2012) se enfocó en analizar las diferencias de edad y sexo a partir del instrumento BALLI con una población de 64 hombres y 136 mujeres. Los resultados indicaron que para las mujeres la habilidad de hablar varios idiomas tiene que ver con la inteligencia y, en mayor porcentaje, manifestaron tener más habilidad que los hombres para aprender una lengua extranjera. Además manifestaron que las habilidades de hablar y escuchar son las más fáciles y que la gramática es la parte más importante cuando se aprende un nuevo idioma.

Mudra (2016) analizó las diferencias entre ambos sexos en el contexto universitario en Indonesia, con una población de 54 mujeres y 46 hombres. Los hallazgos mostraron que en mayor proporción las mujeres confirmaron estar de acuerdo con que las personas que son buenas en matemáticas y ciencias no tienen la habilidad para aprender una lengua extranjera. Igualmente se encontró que en mayor porcentaje las mujeres consideraron que aprenderían a hablar inglés de manera satisfactoria en el contexto en donde lo están aprendiendo, mientras que los hombres se mostraron pesimistas al respecto.

\section{Marco metodológico}

La metodología de esta investigación está tipificada como descriptiva, dado que describe las diferencias en las creencias de hombres y mujeres acerca del aprendizaje del idioma inglés. Bernal (2016: 143) define la investigación descriptiva como aquella en donde "se muestran, narran, reseñan o identifican hechos, situaciones, rasgos, características de un objeto de estudio". Se estableció un diseño de campo y transeccional descriptivo, debido que "la información del objeto de estudio se obtiene en una única vez en un momento dado" (Bernal, 2016: 145). Se considera que es de campo por haberse observado en el contexto del Tecnológico de Antioquia Institución Universitaria. Se establece no experimental en virtud de que no se pretende alterar las creencias como variable central del estudio. 
Se empleó el censo poblacional, al considerar el total de los individuos; posteriormente se establecieron dos estratos poblacionales en correspondencia con la variable sexo, donde el primero quedó conformado por 35 mujeres y el segundo por 131 hombres, los cuales cursan la asignatura de inglés en los programas técnicos, tecnológicos y profesionales, en los niveles I a VI. Se aclara que todos los estudiantes participantes del estudio han estudiado inglés siguiendo el mismo currículo propuesto por el Área de Lengua extranjera del Tecnológico de Antioquia - Institución Universitaria. Esta institución educativa es de carácter público, opera como ente descentralizado de la Gobernación de Antioquia en la ciudad de Medellín, departamento de Antioquia, Colombia; y alberga estudiantes de estratos sociales bajo $(1,2)$ y medio (3). El nivel de conocimiento del idioma de los participantes está entre los niveles A1y B1, de acuerdo con el Marco Común de Referencia para las Lenguas. Ninguno ha estudiado un idioma diferente al inglés y el método de enseñanza corresponde al enfoque comunicativo y al aprendizaje basado en tareas y estrategias (Consejo de Europa, 2001).

Se utilizó el Instrumento Especializado para la Medición BALLI propuesto por Horwitz (1987), conformado por un total de cinco cuestionarios con un total de (34 ítems) en atención a las cinco dimensiones que lo conforman. Los cuestionarios fueron traducidos al idioma español para facilitar la comprensión de cada ítem, y posteriormente fueron presentados a los estudiantes. Un lingüista experimentado revisó la traducción de cada ítem, aunque se efectuaron algunas adaptaciones para ajustarlas al contexto del estudio. Las palabras Some languages (Balli\#3), se reemplazaron por las palabras 'por aprender inglés' (ítem 9); la creencia Balli\# 6 people from my country, se cambió por 'las personas de Colombia' (ítem 3); la palabra Americans (Balli\#13), se cambió por 'otros colombianos' (ítem 23); en la aseveración Balli \#15 el tiempo estimado está en los rangos comprendidos entre 1 año y 10 años, y se reemplazó por un margen entre 1 año y 5 (ítem 11). Se incluyó el ítem 20 (¿la estructura del idioma español es diferente a la del inglés?) en lugar del enunciado Balli \#16 I have a special ability for learning foreing languages. La frase people in my country (Balli \#20), se reemplazó por 'las personas en Colombia' (ítem 30); las palabras cassettes or tapes (Balli \#26), se cambiaron por 'grabaciones o videos' (ítem 28); las palabras native language (Balli \#28), se reemplazaron por 'español' (ítem 19).

Con relación a los índices de confiabilidad del instrumento BALLI, el coeficiente obtenido para dicho inventario de creencias fue de 0,598 realizado a través del test de confiabilidad Alfa de Cronbach. De este resultado podría inferirse que las creencias analizadas tienen una naturaleza heterogénea, lo cual es previsible ya que se está hablando de creencias frente al aprendizaje de una lengua extranjera. Esto también podría explicar la falta de correlación entre los coeficientes de cada uno de los ítems con el total de la escala. Al respecto, Khodadady (2009) sostiene que debido a las discrepancias, diversos investigadores han rechazado el análisis de confiabilidad de los 
resultados (Horwitz, 1985; Peacock, 2001) y que de hecho, ningún estudio realizado con el instrumento BALLI ha reportado tener alguna correlación entre las aseveraciones que contiene cada ítem. Se realizó la revisión de la literatura frente a la aplicación del instrumento BALLI en estudios en el contexto latinoamericano pero no se encontró evidencia de cálculos de confiabilidad.

Cada ítem fue categorizado a partir de la escala de Likert de la siguiente manera: 1 totalmente de acuerdo (TA), 2 de acuerdo (A), 3 neutral (N), 4 en desacuerdo (D) y 5 totalmente en desacuerdo (TD). Con base en la participación de las respuestas en cada una de estas escalas, se realizó una agrupación de las categorías TA con A, y D con TD, dado que estos extremos concentraron el mayor número de datos; las respuestas neutrales no se tuvieron en cuenta por su baja frecuencia. Los datos fueron presentados en cinco tablas en forma de porcentajes en cada una de las categorías de acuerdo a la cantidad de personas en cada sexo.

Los datos obtenidos tras la aplicación del instrumento fueron analizados con estadística descriptiva e inferencial empleando el paquete estadístico SPSS versión 23, para analizar la información y obtener datos precisos. Se aplicó la prueba de normalidad Kolmogorov-Smirnov y esta indicó que los datos no siguen una distribución normal ya que el nivel de significancia fue de 0,000 para todos los ítems. Por tratarse de dos estratos independientes con datos presentados en una escala ordinal, se contrastó el comportamiento en ambas poblaciones mediante la prueba no paramétrica U de Mann-Whitney. Esta es una prueba de dos colas, de la forma: $\mathrm{H}_{0}$ : $\mathbf{M}_{1}=\mathbf{M}_{\mathbf{2}} ; \mathrm{H}_{1:} \mathbf{M}_{\mathbf{1}} \neq \mathbf{M}_{\mathbf{2}}$. Dicho test fue empleado para comprobar posibles diferencias entre las creencias de los hombres y las mujeres en cada uno de los ítems y se definió un nivel de significancia de alfa $(\alpha)$ del $0,05 \%$.

\section{Resultados}

\subsection{Aptitud para las lenguas extranjeras}

En la primera dimensión se analizaron los ítems 1 a 8 (Tabla 1). En cuanto al ítem 1, el 69,5\% de los hombres y el 85,2\% de las mujeres manifestaron estar de acuerdo. En el ítem 2 el 71,8\% de los hombres y el 69,6\% de las mujeres estuvieron de acuerdo. En lo concerniente al ítem 3, el 40,5\% de los hombres y el 31,9\% de las mujeres expresaron estar de acuerdo. En cuanto a la creencia correspondiente al ítem 4, el $59,5 \%$ de los hombres y el 57,0\% optaron por estar de acuerdo. El 16\% de los hombres y el 8,9\% de las mujeres se inclinaron por estar de acuerdo con el ítem 5 . Un alto porcentaje de hombres $(57,3 \%)$ y mujeres $(68,1 \%)$, definieron estar en desacuerdo. En el ítem 6, el 46,6\% de los hombres y el 54,8\% de las mujeres se identificó con la opción de desacuerdo, mientras que el 34,4\% de los hombres y el $29,6 \%$ de las mujeres estuvieron de acuerdo. En lo referente al ítem 7, una proporción del $34,4 \%$ de los hombres y un $29,6 \%$ de las mujeres definieron estar de acuerdo, 
mientras que un $24,4 \%$ de los hombres y un 33,3\% de las mujeres escogieron la opción de desacuerdo. En lo correspondiente al ítem 8, un 80,2\% de los hombres y un $73,3 \%$ de las mujeres eligieron estar de acuerdo.

Tabla 1. Aptitud para las lenguas extranjeras.

\begin{tabular}{|c|c|c|c|c|c|c|}
\hline Ítems & Sexo & N (TA) & $\mathbf{N}(\mathrm{A})$ & $\mathbf{N}(\mathbf{N})$ & $\mathbf{N}(\mathrm{D})$ & $\mathbf{N}$ (TD) \\
\hline \multirow{2}{*}{$\begin{array}{l}\text { 1. ¿Es más fácil para los niños aprender una } \\
\text { lengua extranjera que para los adultos? }\end{array}$} & M & $43,5 \%$ & $26,0 \%$ & $22,9 \%$ & $5,3 \%$ & $2,3 \%$ \\
\hline & $\mathrm{F}$ & $55,6 \%$ & $29,6 \%$ & $11,9 \%$ & $2,2 \%$ & $7 \%$ \\
\hline \multirow{2}{*}{$\begin{array}{l}\text { 2. ¿Algunas personas tienen una habilidad } \\
\text { especial para las lenguas extranjeras? }\end{array}$} & M & $29,0 \%$ & $42,7 \%$ & $17,6 \%$ & $7,6 \%$ & $1 \%$ \\
\hline & $\mathrm{F}$ & $26,7 \%$ & $43,0 \%$ & $22,2 \%$ & $8,1 \%$ & $0,0 \%$ \\
\hline \multirow{2}{*}{$\begin{array}{l}\text { 3. ¿Las personas de Colombia son buenas para } \\
\text { aprender una lengua extranjera? }\end{array}$} & $\mathrm{M}$ & $10,7 \%$ & $29,8 \%$ & $48,9 \%$ & $9,2 \%$ & $1,5 \%$ \\
\hline & $\mathrm{F}$ & $8,1 \%$ & $23,7 \%$ & $54,1 \%$ & $11,9 \%$ & $2,2 \%$ \\
\hline \multirow{2}{*}{$\begin{array}{l}\text { 4. ¿Es más fácil para una persona que ya habla } \\
\text { una lengua extranjera aprender otra? }\end{array}$} & $\mathrm{M}$ & $28,2 \%$ & $31,3 \%$ & $30,5 \%$ & $8,4 \%$ & $1,5 \%$ \\
\hline & $\mathrm{F}$ & $18,5 \%$ & $38,5 \%$ & $30,4 \%$ & $11,9 \%$ & $0,7 \%$ \\
\hline \multirow{2}{*}{$\begin{array}{l}\text { 5. ¿Las personas que son buenas en } \\
\text { matemáticas o ciencias no son buenas para } \\
\text { aprender una lengua extranjera? }\end{array}$} & M & $6,1 \%$ & $9,9 \%$ & $26,7 \%$ & $40,5 \%$ & 16,8 \\
\hline & $\mathrm{F}$ & 2,2 & $6,7 \%$ & $23,0 \%$ & $52,6 \%$ & $15,6 \%$ \\
\hline \multirow{2}{*}{$\begin{array}{l}\text { 6. ¿Las mujeres son mejores que los hombres } \\
\text { para los idiomas? }\end{array}$} & $\mathrm{M}$ & $1,5 \%$ & $6,9 \%$ & $45,0 \%$ & $22,9 \%$ & $23,7 \%$ \\
\hline & $\mathrm{F}$ & $2,2 \%$ & $3,7 \%$ & $39,3 \%$ & $35,6 \%$ & $19,3 \%$ \\
\hline \multirow{2}{*}{$\begin{array}{l}\text { 7. ¿Las personas que hablan más de un idioma } \\
\text { son muy inteligentes? }\end{array}$} & $\bar{M}$ & $9,2 \%$ & $25,2 \%$ & $41,2 \%$ & $15,3 \%$ & $9,2 \%$ \\
\hline & $\mathrm{F}$ & $5,9 \%$ & $23,7 \%$ & $37,0 \%$ & $26,7 \%$ & $6,7 \%$ \\
\hline \multirow{2}{*}{$\begin{array}{l}\text { 8. ¿Todas las personas tienen la habilidad de } \\
\text { aprender a hablar una lengua extranjera? }\end{array}$} & $\mathrm{M}$ & $48,9 \%$ & $31,3 \%$ & $13,0 \%$ & $6,1 \%$ & $0,8 \%$ \\
\hline & $\mathrm{F}$ & $34,1 \%$ & $39,3 \%$ & $19,3 \%$ & $6,7 \%$ & $0,7 \%$ \\
\hline
\end{tabular}

La prueba no paramétrica U de Mann-Whitney indicó que no existen diferencias significativas entre las creencias de ambos sexos frente al aprendizaje del inglés en los ítems 2, 3, 4, 5, 6 y 7. En cuanto a los ítems 1 y 8, se encontraron diferencias entre hombres y mujeres (Tabla 2). En el ítem 1, la prueba arrojó un resultado de 7296,500 con P-valor $=0,008$ y en el ítem 8, el resultado fue 7519,000 con P-valor $=0,025$.

Tabla 2. Prueba U de Mann-Whitney, primera dimensión BALLI.

\begin{tabular}{|l|l|l|l|l|l|l|l|l|}
\hline & Ítem 1 & Ítem 2 & Ítem 3 & Ítem 4 & Ítem 5 & Ítem 6 & Ítem 7 & Ítem 8 \\
\hline Mann-Whitney U & 7296,500 & 8666,000 & 7973,000 & 8108,500 & 7971,500 & 8420,000 & 8076,000 & 7519,000 \\
\hline Wilcoxon W & 16476,500 & 17312,000 & 16619,000 & 16754,500 & 16617,500 & 17066,000 & 16722,000 & 16165,000 \\
\hline Z & $-2,672$ &,- 298 & $-1,510$ & $-1,223$ & $-1,481$ &,- 714 & $-1,277$ & $-2,248$ \\
\hline $\begin{array}{l}\text { Asymp. Sig. (2- } \\
\text { tailed) }\end{array}$ &, 008 &, 766 &, 131 &, 221 &, 139 &, 475 &, 202 &, 025 \\
\hline
\end{tabular}

\subsection{La dificultad del aprendizaje de un idioma extranjero}

Los ítems 9 a 13 se enfocaron en el análisis de las creencias con relación a la dificultad del aprendizaje de una lengua extranjera (Tabla 3). Con relación al ítem 9, el $34,4 \%$ de los hombres y 26,7\% de las mujeres declaró estar de acuerdo y un 42,7\% de los hombres y $51,1 \%$ de las mujeres asumió una postura neutral. En cuanto a ítem 10, el 39,3\% de las mujeres lo consideró difícil, mientras que un 39,7\% de los hombres lo consideró fácil. Un $41,2 \%$ de los hombres y un 38,5\% de las mujeres se mostraron optimistas frente al tiempo que le toma a una persona hablar inglés de manera fluida, ya que ambos géneros optaron por pensar que es entre 1 y 2 años. Un 22,9\% de los 
hombres y un 18,5\% de las mujeres optaron por la opción de 3 años. Un 35,9\% de los hombres y un $40 \%$ de las mujeres eligieron estar de acuerdo con que el tiempo es entre 4 y 5 años. Frente al ítem 12, un 51,9\% de los hombres y un 49,6\% de las mujeres estuvieron de acuerdo. Un 14,5\% de los hombres y un $20 \%$ de las mujeres se inclinaron por estar en desacuerdo. En lo concerniente al ítem 13, un 38,9\% de los hombres y un $52,6 \%$ de las mujeres declararon estar de acuerdo, mientras que un el $24,4 \%$ de los hombres y un $20 \%$ de las mujeres manifestaron su desacuerdo con relación a este ítem.

Tabla 3. La dificultad del aprendizaje de un idioma extranjero.

\begin{tabular}{|c|c|c|c|c|c|c|}
\hline ter & Sexo & N (TA) & $\mathbf{N}(\mathbf{A})$ & $\mathbf{N}(\mathbf{N})$ & $\mathbf{N}(\mathbf{D})$ & N (TD) \\
\hline \multirow{2}{*}{$\begin{array}{l}\text { 9. ¿Aprender inglés es más fácil que aprender } \\
\text { otros idiomas? }\end{array}$} & $\mathrm{M}$ & 4 & & $2,7 \%$ & $0,6 \%$ & $2,3 \%$ \\
\hline & $\mathrm{F}$ & & & & & $\%$ \\
\hline \multirow[t]{2}{*}{ 10. ¿El inglés es un idioma difícil de aprender? } & $\mathrm{M}$ & 4, &, $6 \%$ & & $\%$ & $4 \%$ \\
\hline & $\mathrm{F}$ & 14,8 & 24, & 34 & 21 , & $4,4 \%$ \\
\hline \multirow{2}{*}{$\begin{array}{l}\text { 11. ¿Cuánto tiempo le toma a una persona } \\
\text { hablar inglés de manera fluida? TA: } 1 \text { año - A: } \\
2 \text { años }-\mathrm{N}: 3 \text { años - D: } 4 \text { años - TD: } 5 \text { años }\end{array}$} & M & 19 & 22 & 22 & $10,7 \%$ & $\left.25,2^{\circ}\right\rangle$ \\
\hline & $\mathrm{F}$ & 28 & 1 & & 12 & 30 \\
\hline \multirow{2}{*}{$\begin{array}{l}\text { 12. ¿Es más fácil hablar, que comprender lo } \\
\text { que se escucha en inglés? }\end{array}$} & $\mathrm{M}$ & $17,6 \%$ & $34,4 \%$ & $33,6 \%$ & $9,9 \%$ & $4,6 \%$ \\
\hline & $\bar{F}$ & $1,9 \%$ & $37,8 \%$ & $30,4 \%$ & $17,0 \%$ & $3,0 \%$ \\
\hline \multirow{2}{*}{$\begin{array}{l}\text { 13. ¿Es más fácil leer y escribir en inglés que } \\
\text { hablarlo y entenderlo? }\end{array}$} & $\bar{M}$ & 16,0 & 22 & & $17,6 \%$ & $6,9 \%$ \\
\hline & $\mathrm{F}$ & $15,6^{\circ}$ & 37,0 & $27,4 \%$ & $17,0 \%$ & $3,0 \%$ \\
\hline
\end{tabular}

La prueba U de Mann-Whitney, reveló que no existen diferencias significativas entre las creencias de los hombres y las mujeres con relación a los ítems 9, 11, 12 y 13. Respecto al ítem 10, se halló diferencia entre ambos sexos puesto que el resultado de la prueba fue 6974,000 y P-valor $=0,002<0,05$ (Tabla 4).

Tabla 4. Prueba U de Mann-Whitney, segunda dimensión BALLI.

\begin{tabular}{|l|c|c|c|c|c|}
\hline & Ítem 9 & Ítem 10 & Ítem 11 & Ítem 12 & Ítem 13 \\
\hline Mann-Whitney U & 8175,000 & 6974,000 & 8393,500 & 8253,000 & 7843,000 \\
\hline Wilcoxon W & 16821,000 & 16154,000 & 17039,500 & 16899,000 & 17023,000 \\
\hline Z & $-1,136$ & $-3,095$ &,- 734 &,- 983 & $-1,651$ \\
\hline Asymp. Sig. (2-tailed) &, 256 &, 002 &, 463 &, 326 &, 099 \\
\hline
\end{tabular}

\subsection{La naturaleza del aprendizaje de un idioma}

En la Tabla 5 se presentan siete ítems correspondientes a la dimensión de la naturaleza del aprendizaje de un idioma. En consideración con el ítem 14, un 40,5\% de los hombres y un 30,4\% de las mujeres se mostraron de acuerdo. Un 90,1\% de los hombres y un $81,5 \%$ de las mujeres estuvieron de acuerdo con el ítem 15 '. En cuanto al ítem 16 , un $74,8 \%$ de los hombres y un $84,4 \%$ de las mujeres se identificaron con dicha creencia. Frente a la creencia enunciada en el ítem 17, un 53,4\% de los hombres y un $58,5 \%$ de las mujeres manifestaron estar de acuerdo. Con relación al ítem 18, un $61,1 \%$ de los hombres y un $70,4 \%$ de las mujeres declararon estar de acuerdo. Respecto a la creencia correspondiente al ítem 19, un 35,9\% de los hombres y un 37\% de las mujeres sustentaron estar de acuerdo. Un 33,6\% de los hombres y un 34,1\% de 
las mujeres no estuvieron de acuerdo con dicha afirmación. Finalmente, un 80,9\% de los hombres y un $90,4 \%$ de las mujeres se mostraron convencidos en cuanto a que 'La estructura del idioma español es diferente a la del inglés'.

Tabla 5. La naturaleza del aprendizaje de un idioma.

\begin{tabular}{|c|c|c|c|c|c|c|}
\hline Ítem & Sexo & N (TA) & $\mathbf{N}(\mathrm{A})$ & $\mathbf{N}(\mathbf{N})$ & $\mathbf{N}(\mathrm{D})$ & $\mathbf{N}$ (TD) \\
\hline \multirow{2}{*}{$\begin{array}{l}\text { 14. ¿Es necesario aprender sobre las culturas de } \\
\text { habla inglesa para aprender a hablar inglés? }\end{array}$} & $M$ & $12,2 \%$ & $28,2 \%$ & $30,5 \%$ & $24,4 \%$ & $4,6 \%$ \\
\hline & $\mathrm{F}$ & & &, $1 \%$ & & \\
\hline \multirow{2}{*}{$\begin{array}{l}\text { 15. ¿Es mejor aprender inglés en un país de } \\
\text { habla inglesa? }\end{array}$} & $\mathrm{M}$ & & & $\%$ & & $\%$ \\
\hline & $\mathrm{F}$ & & $\%$ & 0 & & $0,0 \%$ \\
\hline \multirow{2}{*}{$\begin{array}{l}\text { 16. ¿La parte más importante del aprendizaje de } \\
\text { una lengua extranjera es el vocabulario? }\end{array}$} & $\mathrm{M}$ & 39 & $35,1 \%$ & $\%$ & & 0,0 \\
\hline & $\mathrm{F}$ & 32 , & $51,9 \%$ & $\%$ & $\%$ & $0,0 \%$ \\
\hline \multirow{2}{*}{$\begin{array}{l}\text { 17. ¿La parte más importante del aprendizaje de } \\
\text { una lengua extranjera es la gramática? }\end{array}$} & $\mathrm{M}$ & & $36,6 \%$ & & $5,3 \%$ & $2,3 \%$ \\
\hline & $\mathrm{F}$ & & $45,2 \%$ & $34,1 \%$ & $7,4 \%$ & $0,0 \%$ \\
\hline \multirow{2}{*}{$\begin{array}{l}\text { 18. ¿Aprender una lengua extranjera es } \\
\text { diferente a aprender otra asignatura? }\end{array}$} & $\mathrm{M}$ & & $35,9 \%$ &, $7 \%$ & $11,5 \%$ & $3,8 \%$ \\
\hline & $\mathrm{F}$ & & $15,9 \%$ & & & $2,2 \%$ \\
\hline \multirow{2}{*}{$\begin{array}{l}\text { 19. ¿La parte más importante del aprendizaje } \\
\text { del inglés es aprender a traducir desde el } \\
\text { español? }\end{array}$} & $\mathrm{M}$ & 0 & $27,5 \%$ & $30,5 \%$ & $\%$ & $14,5 \%$ \\
\hline & F & 7 & $29,6 \%$ & $28,9 \%$ & $28,1 \%$ & 5,9 \\
\hline \multirow{2}{*}{$\begin{array}{l}\text { 20. ¿La estructura del idioma español es } \\
\text { diferente a la del inglés? }\end{array}$} & $\bar{M}$ & & $41,2 \%$ & & & $3,1 \%$ \\
\hline & $\mathrm{F}$ & $50,4 \%$ & $40,0 \%$ & $8,1 \%$ & $1,5 \%$ & $0,0 \%$ \\
\hline
\end{tabular}

La prueba U de Mann-Whitney, indicó que no hay diferencias significativas entre las creencias de los hombres y las mujeres en los ítems 14, 16, 17, 18 y 19. Con relación a los ítems 15 y 20, se descubrieron diferencias entre ambos sexos, ya que en el ítem 15 el resultado de la prueba fue 7350,000, con un P-valor $=0,009<0,05$ y en el ítem 20, el P-valor $=0,024<0,05$, con un resultado de 7543,500 (Tabla 6).

Tabla 6. Prueba U de Mann-Whitney, tercera dimensión BALLI.

\begin{tabular}{|l|c|c|c|c|c|c|c|}
\hline & Ítem 14 & Ítem 15 & Ítem 16 & Ítem 17 & Ítem 18 & Ítem 19 & Ítem 20 \\
\hline Mann-Whitney U & 7820,000 & 7350,000 & 8752,000 & 8642,000 & 8403,500 & 8581,000 & 7543,500 \\
\hline Wilcoxon W & 16466,000 & 15996,000 & 17932,000 & 17822,000 & 17583,500 & 17761,000 & 16723,500 \\
\hline Z & $-1,691$ & $-2,612$ &,- 155 &,- 341 &,- 735 &,- 431 & $-2,259$ \\
\hline Asymp. Sig. (2-tailed) &, 091 &, 009 &, 877 &, 733 &, 463 &, 666 &, 024 \\
\hline
\end{tabular}

\subsection{Las estrategias de aprendizaje y comunicación}

En los ítems 21 a 28 se examinaron las creencias de los encuestados respecto a las estrategias de aprendizaje y comunicación (Tabla 7). En lo que respecta al ítem 21, un $80,9 \%$ de los hombres y un $85,2 \%$ de las mujeres optaron por estar de acuerdo. En lo referente al ítem 22, un 67,9\% de los hombres y un $69,6 \%$ de las mujeres afirmaron estar en desacuerdo. Con relación a la creencia correspondiente al ítem 23, un 61,1\% de los hombres y un 58,5\% de las mujeres coincidieron en estar de acuerdo. Un 52,7\% de los hombres encuestados y un $57 \%$ de las mujeres, manifestaron estar en desacuerdo con el ítem 24. En lo concerniente al ítem 25, un 94,7\% de los hombres y un $94,8 \%$ de las mujeres respaldaron la opción de estar de acuerdo. Frente a la creencia presentada en el ítem 26, un 21,4\% de los hombres y un 46,7\% de las mujeres 
indicaron estar de acuerdo. Con relación al ítem 27, un 35,9\% de los hombres y un $51,1 \%$ de las mujeres confirmaron estar de acuerdo. Finalmente, con relación al ítem 28 , un $65,6 \%$ de los hombres y un 78,5\% de las mujeres definieron estar de acuerdo.

Tabla 7. Las estrategias de aprendizaje y comunicación.

\begin{tabular}{|c|c|c|c|c|c|c|}
\hline Ítem & Sexo & $\mathbf{N}$ (TA) & $\mathbf{N}(\mathrm{A})$ & $\mathbf{N}(\mathbf{N})$ & $\mathbf{N}(\mathrm{D})$ & $\mathbf{N}$ (TD) \\
\hline \multirow{2}{*}{$\begin{array}{l}\text { 21. ¿Es importante hablar inglés con una } \\
\text { pronunciación excelente? }\end{array}$} & $\mathrm{M}$ & $43,5 \%$ & $37,4 \%$ & $3,7 \%$ & $3,1 \%$ & $2,3 \%$ \\
\hline & F & $+\%$ & $\%$ & $\%$ & $2,2 \%$ & $0,0 \%$ \\
\hline \multirow{2}{*}{$\begin{array}{l}\text { 22. ¿Usted no debería decir nada en inglés hasta } \\
\text { que no pueda decirlo correctamente? }\end{array}$} & $\mathrm{M}$ & $5,3 \%$ & $9,2 \%$ & $17,6 \%$ & $41,2 \%$ & $26,7 \%$ \\
\hline & $\mathrm{F}$ & $3,0 \%$ & $11,9 \%$ & $15,6 \%$ & $54,1 \%$ & $15,6 \%$ \\
\hline \multirow{2}{*}{$\begin{array}{l}\text { 23. ¿Disfruto practicar inglés con otros } \\
\text { "colombianos"? }\end{array}$} & $\mathrm{M}$ & $206 \%$ & $40,5 \%$ & & $3,8 \%$ & $2,3 \%$ \\
\hline & $\mathrm{F}$ & $0,4 \%$ & $48,1 \%$ & 31 & $7,4 \%$ & $3,0 \%$ \\
\hline \multirow{2}{*}{$\begin{array}{l}\text { 24. ¿Es adecuado adivinar si usted no sabe una } \\
\text { palabra en inglés? }\end{array}$} & $\mathrm{M}$ & $3 \%$ & $12,2 \%$ & $\%$ & $33,6 \%$ & $19,1 \%$ \\
\hline & $\mathrm{F}$ & 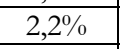 & $\%$ & $\%$ & $43,7 \%$ & $13,3 \%$ \\
\hline \multirow{2}{*}{$\begin{array}{l}\text { 25. ¿Es importante repetir y practicar mucho } \\
\text { para aprender inglés? }\end{array}$} & $\mathrm{M}$ & 0 & 8 & & $2,3 \%$ & $0,0 \%$ \\
\hline & $\mathrm{F}$ & $68,9 \%$ & $25,9 \%$ & $5,2 \%$ & $0,0 \%$ & $0,0 \%$ \\
\hline \multirow{2}{*}{$\begin{array}{l}\text { 26. ¿Me da vergüenza hablar en inglés con otras } \\
\text { personas? }\end{array}$} & $\mathrm{M}$ & $6,1 \%$ & $15,3 \%$ & $32,8 \%$ & $22,9 \%$ & $22,9 \%$ \\
\hline & $\mathrm{F}$ & $20,0 \%$ & $26,7 \%$ & $25,9 \%$ & $22,2 \%$ & $5,2 \%$ \\
\hline \multirow{2}{*}{$\begin{array}{l}\text { 27. ¿Si a los principiantes se les permite } \\
\text { cometer errores en inglés, será difícil para ellos } \\
\text { hablar correctamente en un futuro? }\end{array}$} & $\mathrm{M}$ & $12,2 \%$ & $23,7 \%$ & $22,9 \%$ & $25,2 \%$ & $16,0 \%$ \\
\hline & $\mathrm{F}$ & 14 & $37,0 \%$ & $11,9 \%$ & $27,4 \%$ & $9,6 \%$ \\
\hline \multirow{2}{*}{$\begin{array}{l}\text { 28. ¿Es importante practicar con grabaciones o } \\
\text { videos? }\end{array}$} & $\bar{M}$ & $29,8 \%$ & $35,9 \%$ & $26,7 \%$ & $4,6 \%$ & $3,1 \%$ \\
\hline & $\mathrm{F}$ & $21,5 \%$ & $57,0 \%$ & $16,3 \%$ & $3,7 \%$ & $1,5 \%$ \\
\hline
\end{tabular}

El análisis de la cuarta dimensión a través de la prueba U de Mann-Whitney, comprobó que no hay diferencias significativas entre las creencias de los hombres y las mujeres en los ítems 21, 22, 23, 24, 25, 27 y 28. Por otro lado, se identificaron diferencias entre ambos sexos en el ítem 26, puesto que el P-valor $=0,000<0,05$ y el resultado de la prueba es de 5880,500 (Tabla 8).

Tabla 8. Prueba U de Mann-Whitney, cuarta dimensión BALLI.

\begin{tabular}{|l|l|l|l|l|l|l|l|l|}
\hline & Ítem 21 & Ítem 22 & Ítem 23 & Ítem 24 & Ítem 25 & Ítem 26 & Ítem 27 & Ítem 28 \\
\hline Mann-Whitney U & 8313,500 & 8283,000 & 982,500 & 8832,00 & 8617,00 & 5880,500 & 7745,00 & 8533,00 \\
\hline Wilcoxon W & 17493,500 & 17463,00 & 16628,50 & 18012,00 & 17263,00 & 15060,50 & 16925,00 & 17713,00 \\
\hline Z &,- 914 &,- 953 & $-1,465$ &,- 017 &,- 450 & $-4,847$ & $-1,801$ &,- 528 \\
\hline $\begin{array}{l}\text { Asymp. Sig. (2- } \\
\text { tailed) }\end{array}$ &, 360 &, 341 &, 143 &, 986 &, 653 &, 000 &, 072 &, 597 \\
\hline
\end{tabular}

\subsection{Motivaciones y expectativas}

En esta dimensión se examinaron los ítems 29 al 34, los cuales se presentan en la Tabla 9. El 86,3\% de los hombres y un 65,9\% de las mujeres pensaron que aprenderán a hablar muy bien en inglés (ítem 29). En relación al ítem 30, un 74\% de los hombres y un 69,6\% de las mujeres coincidieron en estar de acuerdo. En cuanto al ítem 31 , un $74,8 \%$ de los hombres y un $77 \%$ de las mujeres se mostraron de acuerdo. Un $92,4 \%$ de los hombres y un $93,3 \%$ las mujeres optaron por estar de acuerdo con el ítem 32. Un 98,5\% de los hombres y un 97,8\% de las mujeres aseguraron estar de acuerdo con el ítem 33. Un $54,2 \%$ de los hombres y un 43,7\% de las mujeres están interesados en hablar inglés porque les gustaría tener amigos americanos. 
Tabla 9. Motivaciones y expectativas.

\begin{tabular}{|c|c|c|c|c|c|c|}
\hline Ítem & Sexo & N (TA) & $\mathbf{N}(\mathrm{A})$ & $\mathbf{N}(\mathbf{N})$ & $\mathbf{N}(\mathrm{D})$ & $\mathbf{N}$ (TD) \\
\hline \multirow{2}{*}{$\begin{array}{l}\text { 29. ¿Creo que aprenderé a hablar muy bien en } \\
\text { inglés? }\end{array}$} & $\mathrm{M}$ & $46,6 \%$ & $39,7 \%$ & $11,5 \%$ & $2,3 \%$ & $0,0 \%$ \\
\hline & $\mathrm{F}$ & $26,7 \%$ & $39,3 \%$ & $27,4 \%$ & $5,9 \%$ & $0,7 \%$ \\
\hline \multirow{2}{*}{$\begin{array}{l}\text { 30. ¿La gente en Colombia siente que es } \\
\text { importante hablar inglés? }\end{array}$} & $\mathrm{M}$ & $35,9 \%$ & $38,2 \%$ & $19,1 \%$ & $4,6 \%$ & $2,3 \%$ \\
\hline & $\mathrm{F}$ & $27,4 \%$ & $42,2 \%$ & $17,8 \%$ & $11,9 \%$ & $0,7 \%$ \\
\hline \multirow{2}{*}{$\begin{array}{l}\text { 31. ¿A mí me gustaría aprender inglés de } \\
\text { manera que pueda entender la cultura } \\
\text { americana? }\end{array}$} & M & $44,3 \%$ & $30,5 \%$ & $20,6 \%$ & $3,1 \%$ & $1,5 \%$ \\
\hline & F & 37 & 3. & $15,6 \%$ & $5,2 \%$ & $2,2 \%$ \\
\hline \multirow{2}{*}{$\begin{array}{l}\text { 32. ¿Si aprendo inglés muy bien, tendré mejores } \\
\text { oportunidades laborales? }\end{array}$} & $\mathrm{M}$ & $74,8 \%$ & $17,6 \%$ & $6,1 \%$ & $0,8 \%$ & $0,8 \%$ \\
\hline & $\mathrm{F}$ & $73,3 \%$ & $20,0 \%$ & $5,9 \%$ & $0,7 \%$ & $0,0 \%$ \\
\hline \multirow{2}{*}{$\begin{array}{l}\text { 33. ¿Quiero aprender a hablar en inglés muy } \\
\text { bien? }\end{array}$} & $\mathrm{M}$ & $83,2 \%$ & $15,3 \%$ & $1,5 \%$ & $0,0 \%$ & $0,0 \%$ \\
\hline & $\mathrm{F}$ & $77,0 \%$ & $20,7 \%$ & $2,2 \%$ & $0,0 \%$ & $0,0 \%$ \\
\hline \multirow{2}{*}{$\begin{array}{l}\text { 34. ¿Me motiva hablar inglés porque me } \\
\text { gustaría tener amigos americanos? }\end{array}$} & $\mathrm{M}$ & $26,0 \%$ & $28,2 \%$ & $33,6 \%$ & $9,9 \%$ & $2,3 \%$ \\
\hline & $\mathrm{F}$ & $13,3 \%$ & $30,4 \%$ & $37,0 \%$ & $13,3 \%$ & $5,9 \%$ \\
\hline
\end{tabular}

En lo concerniente a los ítems 30,31, 32 y 33 (Tabla 10), se observó que no hay diferencias significativas entre las creencias de ambos sexos. Se presentaron diferencias en los ítems 29 y 34, ya que en el ítem 29 el P-valor $=0,000<0,05$ con un resultado de fue de 6350,500 y el ítem 34 tuvo un resultado en la prueba de 7353,500 con un Pvalor $=0,013<0,05$ (tabla 10$)$.

Tabla 10. Prueba U de Mann-Whitney, quinta dimensión BALLI.

\begin{tabular}{|l|c|c|c|c|c|c|}
\hline & Ítem 29 & Ítem 30 & Ítem 31 & Ítem 32 & Ítem 33 & Ítem 34 \\
\hline Mann-Whitney U & 6350,500 & 7954,000 & 8451,500 & 8748,000 & 8295,000 & 7353,500 \\
\hline Wilcoxon W & 14996,500 & 16600,000 & 17097,500 & 17394,000 & 16941,000 & 15999,500 \\
\hline Z & $-4,229$ & $-1,496$ &,- 664 &,- 197 & $-1,259$ & $-2,473$ \\
\hline Asymp. Sig. (2-tailed) &, 000 &, 135 &, 507 &, 844 &, 208 &, 013 \\
\hline
\end{tabular}

\section{Discusión de los resultados}

Horwitz (1999) comparó las creencias sobre el aprendizaje del inglés con grupos de estudiantes de Francia, Japón, Turquía, Corea y Taiwán, y concluyó que, a pesar de las diferencias culturales, estilos de aprendizaje, tipo de instrucción y características individuales, estas poblaciones tienen afinidades en las mismas creencias. Por lo anterior, podría inferirse que, pese a las variaciones culturales entre el contexto de Colombia y los países previamente mencionados, habría similitudes en las creencias que tienen ambos grupos. Sin embargo, no se han encontrado estudios que comprueben tal inferencia.

Este estudio indicó que una mayor cantidad de mujeres está de acuerdo con la creencia 'para los niños es más fácil aprender una lengua extranjera'. Dicho resultado es consistente con las ideas de Mudra (2016), pero se encuentra en oposición con Bernat y Lloyd (2007), Rieger (2009) y Daif-Allah (2012). Para un porcentaje significativo de hombres, todas las personas tienen la habilidad de aprender una lengua extranjera; no obstante, esto se encuentra distante de los resultados de Bernat y Lloyd 
(2007), Rieger (2009), Daif-Allah, (2012) y Mudra (2016). Además, se encontró que para ambos sexos la habilidad de aprender varios idiomas no tiene que ver con la inteligencia, lo cual difiere de los resultados de Barkhordar y Rastegar (2012).

Los hombres respaldaron que el contexto adecuado para aprender inglés es un país de habla inglesa; este hallazgo es congruente con Daif-Allah (2012) y Mudra (2016), aunque discrepa con Bernat y Lloyd (2007) y Barkhordar y Rastegar (2012). Al respecto, podría suponerse que esta apreciación tiene su fundamento en la carencia de oportunidades de los estudiantes para interactuar con hablantes nativos en el país en donde se realizó el estudio. En cuanto a la estructura del idioma, no se encontró otro estudio en el que se incluyera la creencia sobre la diferencia entre la tipología sintáctica del español y la del inglés. Para este caso, esta investigación reveló que hay una mayor cantidad de mujeres con esta creencia. Podría también afirmarse que los hombres que estuvieron de acuerdo también ignoran que el idioma inglés y el español están regidos por la misma tipología sintáctica (S-V-O), lo que es confirmado por Villanueva (2011).

Frente a la dimensión de la dificultad del idioma, los resultados porcentuales fueron similares en ambos sexos, a excepción de uno. Para este caso, en mayor proporción, las mujeres reportaron que el inglés es un idioma difícil de aprender, lo cual es congruente con Mudra (2016), aunque difiere de los resultados de Bernat y Lloyd (2007) y Daif-Allah (2012).

En lo referente a las estrategias de comunicación, se descubrió que las mujeres sienten mayor temor para comunicarse en inglés con otras personas. Este aspecto se encuentra en concordancia con Mudra (2016). Por otro lado, ambos sexos reconocieron la repetición y la práctica continua como estrategias útiles, aunque desecharon la estrategia de adivinar palabras a partir de un contexto. Esto es congruente con Mudra (2016) pero discrepa del estudio de Daif-Allah (2012).

El análisis de la dimensión de las motivaciones y expectativas reveló que ambos géneros poseen interés en adquirir la competencia comunicativa, ya que esta les permitirá comunicarse con otros hablantes en otros países y acceder a oportunidades laborales. No obstante, los hombres se mostraron más optimistas sobre esta capacidad, aunque esto discrepa con los hallazgos de Bernat y Lloyd (2007) y (Mudra, 2016) pero se encuentra alineado con Daif-Allah (2012). Pese a que los resultados indicaron que hay una alta motivación en ambos géneros, esto no indica que se dedique suficiente tiempo y esfuerzo para el aprendizaje de este idioma. Adicionalmente, se presentó una diferencia frente al deseo que tienen los participantes de adquirir la competencia comunicativa para tener oportunidades de conocer e interactuar con amigos extranjeros que hablen inglés. Al respecto, los hombres tuvieron una mayor motivación integrativa, lo cual es incongruente con Bernat y Lloyd (2007), Rieger (2009) y Mudra (2016). 
Las ocho creencias en donde se hallaron diferencias significativas representan un $23,5 \%$ con relación a los 34 ítems analizados y comprenden los siguientes porcentajes en cada uno de ellos: ítem 1 (M: 69,5\% - F: 85,2\%); ítem 8 (M: 80,2\% - F: 73,3\%); ítem 10 (M: 25,2\% - F: 39,7\%); ítem 15 (M: 90,1\% - F: 81,5\%); ítem 20 (M: 80,9\% F:90,4\%); ítem 26 (M: 21,4\% - F: 46,7\%); ítem 29 (M: 86,3\% - F: 65,9\%); ítem 34 (M: $54,2 \%$ - F: 43,7\%). Las diferencias encontradas en el contexto del estudio podrían explicarse a partir de ciertos factores, tales como: aspectos biológicos, memoria, habilidad para aprender un idioma extranjero o motivación. Dichos aspectos podrían aclarar, de alguna manera, las razones por las cuales se presentan tales diferencias en la muestra analizada con relación al inventario de creencias BALLI.

En cuanto al aspecto biológico, Burman, Bitan y Booth (2008) sostienen que ciertas áreas del cerebro femenino son activadas cuando se realizan tareas relacionadas con el lenguaje. Por lo tanto, el desempeño de la mujer es mejor que el del hombre. Además, al revisar la anatomía del cerebro humano, la diferencia entre ambos sexos se encuentra en el cuerpo calloso ya que este es, generalmente, más grande en las mujeres. Al respecto, López (2006) revisó diversos estudios en neuropsicología sobre la relación del sexo y el funcionamiento del cerebro y encontró evidencias sobre habilidades superiores de las mujeres para ejecutar tareas referentes al lenguaje oral; en contraste con lo anterior, halló que los hombres se desempeñan mejor en tareas visuales y espaciales. Por esta razón, habría que pensar en el diseño de experiencias de aprendizaje más efectivas que permitan a los hombres mejorar el desempeño en la producción oral a través de actividades que impliquen repeticiones, rimas, lectura en voz alta, juegos de roles, movimiento corporal, entre otras.

Otro aspecto importante frente a las diferencias entre ambos sexos en cuanto al aprendizaje de un idioma, tiene que ver con la memoria. Al respecto, Van Der Slik, Van Hout y Schepens (2015) sustentaron que después de la pubertad, la memoria procedimental se reduce más que la declarativa y este hecho beneficia a las mujeres ya que ellas almacenan la información verbal allí. Esto llevaría a pensar que las mujeres desarrollarían más satisfactoriamente las habilidades de habla y escritura.

En cuanto a la habilidad para aprender un idioma, Sykes (2015) sostiene que la aptitud y la inteligencia son destrezas que están interrelacionadas. Para este investigador, la aptitud contiene aspectos de la inteligencia que son fundamentales para el aprendizaje, pero no sería suficiente para el mismo. En este escenario, sería posible considerar que tanto hombres como mujeres podrían alcanzar diferentes niveles de competencia en una lengua extranjera, dependiendo de ciertos aspectos, como: las características individuales, el contexto (López, 2006) y una metodología que promueva el aprendizaje significativo en términos del logro de la competencia comunicativa. Probablemente, el uso de una prueba de aptitud para lenguas extranjeras como el MLAT (Modern Language Aptitude Test) podría diagnosticar en el 
Tecnológico de Antioquia las características que tiene un individuo para alcanzar cierto nivel de competencia en el idioma inglés y establecer cuál sexo tiene una mayor aptitud. El test en mención mide la habilidad para el aprendizaje por asociación y memorización, la habilidad para el análisis de la gramática y la habilidad para la codificación fonética. Por otro lado, sería interesante probar si el entrenamiento formal con condiciones especiales, en el contexto del estudio, podría de algún modo cuestionar la teoría referente a la inteligencia, independientemente de la variable sexo.

Llama la atención que el resultado de este estudio sea lo opuesto a lo hallado por López (2006), quien después de hacer la revisión de la literatura, concluyó que el éxito de las mujeres en el aprendizaje de un idioma extranjero se debe a que ellas, en parte, muestran una actitud positiva y una motivación integrativa. Mientras que en este estudio se observó una actitud positiva y motivación integrativa, pero en los hombres. Se presume que la explicación de este resultado se debe a que los participantes de este estudio, en particular del sexo masculino, podrían tener aspiraciones relacionadas con el conocimiento de personas extranjeras o de países de habla inglesa.

\section{CONCLUSIONES}

El objetivo planteado en este estudio fue describir las diferencias entre hombres y mujeres acerca del aprendizaje del idioma inglés. Los resultados indicaron que hombres y mujeres tienen creencias similares en consideración con los ítems del inventario de creencias BALLI. No obstante, se evidenciaron diferencias significativas en ocho ítems. Estos hacen referencia a las dimensiones que corresponden a la aptitud, la naturaleza del aprendizaje de un idioma, las estrategias de aprendizaje y comunicación, y la dimensión sobre las motivaciones y expectativas. De hecho, la prueba no paramétrica $U$ de Mann-Whitney demostró que hay diferencias significativas entre hombres y mujeres en los ítems 1, 8, 10, 15, 20, 26, 29 y 34, lo que representa un porcentaje del $23,5 \%$ con relación a las 34 creencias analizadas. Aunque aparentemente es un porcentaje bajo, estas creencias se constituyen en barreras que dificultan el aprendizaje de una lengua extranjera y por tal motivo se considera importante identificarlas. Lo anterior significa que con relación a los hombres, las mujeres sienten mayor inseguridad para comunicarse en inglés con otras personas, piensan que los niños tienen habilidades especiales de las cuales los adultos carecen, conciben el inglés como un idioma difícil de aprender y se inclinan a pensar que la tipología sintáctica del idioma es diferente a la del español. En comparación con las mujeres, los hombres poco confían en que en su país de origen puedan adquirir la competencia comunicativa, sienten una mayor motivación instrumental en cuanto al aprendizaje del inglés y están más seguros de que esta no es una habilidad exclusiva de ciertas personas. A pesar de las diferencias mencionadas, se debe tener en cuenta que hay factores que van más allá del sexo y estos tienen que ver con el contexto, la motivación, los elementos cognitivos, la aptitud, las creencias, la personalidad y los principios afectivos. 
Esta investigación también tiene implicaciones a nivel pedagógico. Primero, es importante que los docentes de idiomas conozcan las creencias que causan un impacto negativo en el aprendizaje y comprendan cómo influyen en el contexto de enseñanza y aprendizaje de una lengua extranjera. Es decir, si un estudiante cree que el aprendizaje del inglés se debe enfocar en el conocimiento de reglas gramaticales, dedicará mayor tiempo a su estudio y esta práctica poco favorecerá el logro de la competencia comunicativa, en concordancia con el Marco Común Europeo de Referencia. En este caso, el docente será un agente que ayudará al estudiante a modificar dicha creencia. Igualmente, si tienen creencias erróneas en cuanto al tiempo que es requerido para alcanzar cierto nivel en una lengua extranjera, este hecho les generará frustración (Cohen, 2010). De la misma manera, el tipo de mensajes que las personas reciben en un contexto determinado frente al aprendizaje de una lengua extranjera, específicamente en el aula de clase, también podría incidir de forma positiva o negativa frente al propósito de alcanzar la competencia esperada.

Segundo, identificar las creencias sobre el aprendizaje de un idioma les permite a los docentes tener mayor cuidado con la selección y creación de material que se utiliza en clase y les posibilita tomar conciencia frente a los procesos que hacen parte del aprendizaje de una lengua extranjera desde una postura psicolingüística y cognitiva. En este sentido, el docente podría proporcionar conocimientos metacognitivos sobre la manera en que se aprende el inglés y brindar herramientas de aprendizaje que faciliten la instrucción, tales como: estrategias cognitivas (repetición de modelos de lenguaje oral y escrito); metacognitivas (planificar la forma de aprender, establecer ritmo de aprendizaje, buscar oportunidades para practicar, verificar el progreso, evaluar la efectividad de las estrategias); afectivas (regular actitudes, reacciones hacia el aprendizaje, auto motivarse y regular la ansiedad); sociales (cooperación con otros estudiantes y búsqueda de oportunidades para usar el idioma); y estrategias de compensación (preguntar cómo se dicen las cosas, usar gestos, usar sinónimos).

Tercero, el conocimiento de las creencias por parte del docente genera una perspectiva diferente frente a los estudiantes que tienen mayores dificultades en el aprendizaje de un idioma. Por ello, es importante que se diseñen espacios de feedback en las clases para fortalecer el proceso de interlenguaje en los estudiantes, el cual está relacionado con: la transferencia lingüística (omisión de pronombres en inglés), transferencia de instrucción (fosilización resultante de una instrucción inadecuada), sobregeneralizaciones de reglas de la L2 (reglas de verbos regulares aplicadas a los irregulares), estrategias de comunicación (hacer parafraseaos y dar ejemplos para compensar la insuficiencia lingüística), y con las estrategias de aprendizaje (traducciones de español a inglés o memorización de estructuras).

Estos resultados conllevan a proponer las siguientes líneas futuras de investigación: realizar comparaciones de diferentes poblaciones considerando las variables de edad, 
estrato socio-económico y nivel de dominio del idioma, e indagar si las creencias de los docentes inciden de alguna manera en el desempeño comunicativo de los estudiantes; analizar si las creencias tienen efecto en los resultados de las pruebas estandarizadas aplicadas para cada uno de los niveles del dominio del idioma, e involucrar diferentes instituciones públicas y privadas de las diferentes regiones del país.

Finalmente, se evidencian algunas limitaciones de esta investigación. Con relación a la variable sexo, las conclusiones de este estudio se consideran parciales ya que sería necesario replicar el estudio con una población mayor de hombres y mujeres. Igualmente, la inclusión de variables como la edad, nivel de competencia en el idioma inglés y estrategias empleadas para el aprendizaje de una lengua extranjera podría ayudar a superar las limitaciones de este trabajo. Por tratarse de una investigación descriptiva no podrían extrapolarse las creencias que tienen ambos sexos sobre el aprendizaje del inglés en un contexto universitario a un contexto de educación básica o media. Por otro lado, la prueba no paramétrica U de Mann-Whitney podría ser complementada con otros métodos para obtener mayor información y enriquecer el análisis de los datos.

\section{REFERENCIAS BIBLIOGRÁFICAS}

Barkhordar, S. \& Rastegar, M. (2012). Effects of gender and grade-level on Iranian English as a foreign language learners' beliefs: Exploring language learning. International Journal of Learning, 18(8), 249-264.

Barrios, E. (2014). Creencias sobre el aprendizaje de una lengua del futuro profesorado de inglés de educación primaria. Porta Linguarum, 22, 73-93.

Bernal, C. (2016). Metodología de la investigación. Administración, Economía, Humanidades y Ciencias Sociales ( $4^{\mathrm{a}}$ ed). Bogotá: Pearson.

Bernat, E. \& Lloyd, R. (2007). Exploring the gender effect on EFL learners' beliefs about language learning. Australian Journal of Educational and Developmental Psychology, 7, 79-91.

Borg, S. (2011). The impact of in-service teacher education on language teachers' beliefs. System, 39(3), 370-380.

Breen, M. P. (2014). Learner contributions to language learning: New directions in research. Nueva York: Routledge.

Burman, D., Bitan, T. \& Booth, J. (2008). Sex differences in neural processing of language among children. Neuropsychologia, 46(5), 1349-1362. 
Cohen, A. D. (2010). Focus on the language learner: Styles, strategies and motivation. En N. Schmitt (Ed.), An Introduction to Applied Linguistics (pp. 161-178). Nueva York: Routledge.

Consejo de Europa (2001). Marco común europeo de referencia para las lenguas: Aprendizaje, enseñaña, evaluación. Madrid: Ministerio de Educación, Cultura y Deporte, Instituto Cervantes.

Daif-Allah, A. S. (2012). Beliefs about foreign language learning and their relationship to gender. English Language Teaching, 5(10), 20-33.

Díaz Larenas, C. \& Moralez Campos, H. (2015). Creencias de estudiantes de primaria sobre el aprendizaje del inglés en un establecimiento educacional chileno. Actualidades Investigativas En Educación, 15(1), 1-20.

Dörnyei, Z. (2005). The Psychology of the language learner. Londres: Lawrence Erlbaum.

Ellis, R. (1994). The study of second language acquisition. Oxford: Oxford University Press.

Gaete, A., Gómez, V. \& Benavides, P. (2017). The overuse of self-report in the study of beliefs in education: Epistemological considerations. International Journal of Research \& Method in Education, 1-16.

Gómez, J. F. (2017). Creencias sobre el aprendizaje de una lengua extranjera en el contexto universitario. Íkala, Revista de Lenguaje y Cultura, 22(2), 203-219.

Horwitz, E. K. (1985). Using student beliefs about language learning and teaching in the foreign language methods course. Foreign Language Annals, 18(4), 333-340.

Horwitz, E. K. (1987). Surveying student beliefs about language learning. En A. Wenden \& J. Rubin (Eds.), Learner strategies in language learning (pp. 119-129). N. J.: Prentice-Hall International.

Horwitz, E. K. (1999). Cultural and situational influences on foreign language learners' beliefs about language learning: A review of BALLI studies. System, 27(4), 557576.

Khodadady, E. (2009). The beliefs about language learning inventory: Factorial validity, formal education and the academic achievement of Iranian students majoring in English. Iranian Journal of Applied Linguistics, 12(1), 115-166.

Loaiza, N. \& Galindo, A. (2014). Estilos de aprendizaje de segundas lenguas y formación bilingüe consecutiva en educación primaria, secundaria y superior: Hacia un estado del arte. Lenguaje, 42(2), 291-314.

López, P. (2006). The sex variable in foreign language learning: An integrative approach. Porta Linguarum, 6(1994), 99-114. 
Mudra, H. (2016). Prospective EFL teachers 'beliefs about language learning and gender differences in a higher education context. International Journal of Academic Research in Education, 2(1), 42-50.

Ormeño, V. \& Rosas, M. (2015). Creencias acerca del aprendizaje de una lengua extranjera en un programa de formación inicial de profesores de inglés en Chile. Colombian Applied Linguistics Journal, 17(2), 207-228.

Oxford, R., Nyikos, M. \& Ehrrnan, M. (1988). Vive la différence? Reflections on sex differences in use of language learning strategies. Foreign Language Annals, 21(4), 321-329.

Peacock, M. (2001). Pre-service ESL teachers' beliefs about second language learning: A longitudinal study. System, 29(2), 177-195.

Phakiti, A. (2003). A closer look at gender and strategy use in L2 reading. Language Learning, 53(4), 649-702.

Radwan, A. A. (2011). Effects of L2 proficiency and gender on choice of language learning strategies by university students majoring in English. Asian EFL Journal, 13(1), 115-163.

Rieger, B. (2009). Hungarian university students' beliefs about language learning: A questionnaire study. $\mathrm{WoPaLP}, 3,97-113$.

San Mateo Valdehíta, A. (2015). Aprendizaje de vocabulario en L2: La relación entre la variable sexo y la efectividad de actividades de reconocimiento y de producción. Resultados preliminares. Epos. Revista de Filología, 31, 27-46.

Sykes, A. H. (2015). The good language learner revisited: A case study. Journal of Language Teaching and Research, 6(4), 713-720.

Van Der Slik, F. W. P., Van Hout, R. W. N. M. \& Schepens, J. J. (2015). The gender gap in second language acquisition: Gender differences in the acquisition of Dutch among immigrants from 88 countries with 49 mother tongues. PLOS ONE, 10(11), 1-22.

Victori, M. \& Lockhart, W. (1995). Enhancing metacognition in self-directed language learning, System, 23(2), 223-234.

Villanueva, A. M. (2011). Effects of the typological distance between languages and of the number of known languages on the acquisition of the syntax of Spanish as a foreign language. Núcleo, 23(28), 217-251.

Zeynali, S. (2012). Exploring the gender effect on EFL learners' learning strategies. Theory and Practice in Language Studies, 2(8), 1614-1620. 


\section{NOTA}

1 Artículo resultado del proyecto de investigación "Creencias de los estudiantes del Tecnológico de Antioquia frente al aprendizaje del inglés como lengua extranjera”, financiado por el Tecnológico de Antioquia - Institución Universitaria, Medellín, Colombia. Centro de costos: 2060001073. 